\title{
Effects of a background axisymmetric potential flow on vortex ring pairing sound
}

\author{
S. K. Tang \\ Department of Building Services Engineering, The Hong Kong Polytechnic University, Hong Kong, \\ People's Republic of China
}

N. W. M. Ko

Department of Mechanical Engineering, The University of Hong Kong, Hong Kong, People's Republic of China

(Received 3 November 1997; revised 14 August 1998; accepted 19 August 1998)

\begin{abstract}
Sound generated by the pairing of two coaxial vortex rings in the presence of a background axisymmetric potential flow was studied numerically. Results show that the background flow substantially affects the sound generation process in the beginning of the vortex ring interaction. They also suggest that the axial jerk and radial acceleration/deceleration of vortex rings are the major mechanisms through which pairing sound is produced. The effect of background flow mean shear rate on sound generation is also discussed and the results suggest that higher sound levels will be generated when the vortex rings are interacting within the region of high mean shear in a low speed laminar jet. (C) 1998 Acoustical Society of America. [S0001-4966(98)06411-X]
\end{abstract}

PACS numbers: 43.28.Ra, 43.28.Py [LCS]

\section{INTRODUCTION}

Powell ${ }^{1}$ suggested that unsteady motion of vorticity is the major source of sound in an unbound low Mach number flow. Although Howe ${ }^{2}$ and Doak ${ }^{3}$ showed that the acoustic variable is the total stagnation enthalpy rather than pressure when vorticity is regarded as the source, the vortex sound theory of Powell ${ }^{1}$ remains valid for low Mach number flow sound generation.

The common vortical structures found within the first four exit diameters of a circular jet are coaxial vortex rings and their pairing is found to be responsible for the growth of the jet mixing layer. ${ }^{4}$ Möhring ${ }^{5}$ solved analytically the sound field generated by two closely packed thin core vortex rings, while Kambe and Minota ${ }^{6}$ extended the investigation numerically to cover vortex rings having the initial core separation comparable to the ring diameter. Results of the latter show that a pulse is generated when the trailing vortex ring shrinks in diameter and slips through the leading ring. The importance of vortex pairing in the development of jet mixing layer and its sound generating characteristics tend to support that vortex ring pairing is a source of noise from the initially laminar low Mach number jets. ${ }^{7}$

Although it is well known that sound is generated by the unsteady motion of vorticity at low Mach number, it remains to be determined at which conditions the loudest noise is likely to be radiated. Investigation into the mechanism of pairing noise generation has been carried out by many researchers (for instance, Ref. 8) and there are experimental results which show that the acceleration and deceleration of vortical structures within the pairing region of a circular jet mixing layer are highly related to the far field jet noise. ${ }^{8,9}$ Leung et al. ${ }^{10}$ modified the vortex ring pairing model of Kambe and Minota ${ }^{6}$ by incorporating the vortex ring formation theory of Saffman ${ }^{11}$ and related explicitly the vortex ring pairing noise with jet noise parameters. There is adequate evidence to conclude that the pairing of vortical ring structures in axisymmetric jets plays a vital role in the noise generation process.

Results of the investigation on the mechanism of thick core vortex ring pairing noise generation of Tang and $\mathrm{Ko}^{12}$ illustrate the importance of the axial jerk and radial acceleration of vortical structures in the generation of sound. However, vortex ring motions in real mixing layers are affected by the background jet mean velocity gradient. This mean velocity gradient, which is also called mean shear rate, may introduce additional jerk and acceleration of vortical structures during the pairing process, making the vortex motions more unsteady, and thus directly change the radiated noise level. The closer the vortex ring to the nozzle lip, the stronger the effect of the mean velocity gradient on pairing noise generation. Therefore, the vortex ring pairing model of Möhring, ${ }^{5}$ Kambe and Minota, ${ }^{6}$ and Leung et al., ${ }^{10}$ while providing a reasonable prediction of jet noise radiation characteristics, may be inadequate in the study of jet noise generation.

In the present study, the effect of a background flow field on pairing noise is studied numerically using a model developed from the results of Leung et al. ${ }^{10}$ and Saffman. ${ }^{11}$ The vortical structure motions, which result in different noise radiation in the presence of the background flow field, are discussed. The causes of the unsteady structure motions are also investigated. The model used in the present study is a simplified one, which may be different from the real shear layer, making comparison between the present results and existing experimental data difficult. However, it is intended here to first investigate the extent the background flow field has on the vortex sound generation and to provide information for future experimental investigations. 


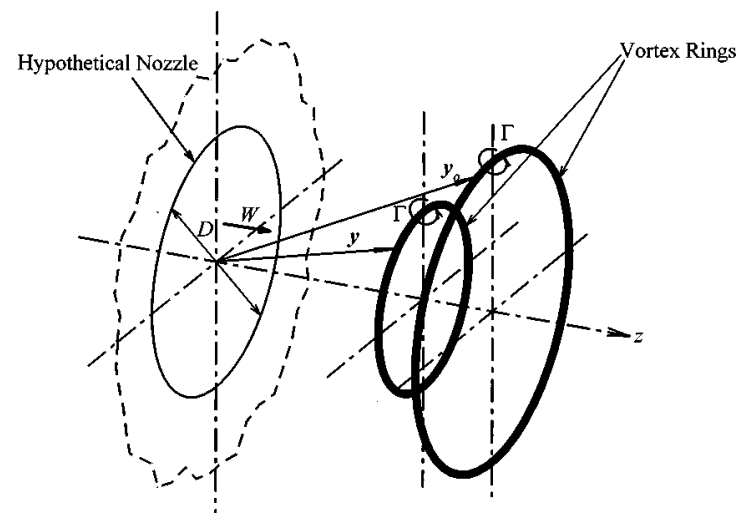

FIG. 1. Schematic diagram of vortex ring system.

\section{THEORETICAL FORMULATION AND COMPUTATIONAL PROCEDURE}

In the present study, the term vortex pairing refers to the mutual threading motion of two coaxial inviscid vortex rings as visualized by Yamada and Matsui. ${ }^{13}$ Noise from vortex coalescence, where the vortex rings merge into a single vortex ring during their mutual threading process ${ }^{13}$ is not studied. It is assumed in the present investigation that the cores of the vortex rings remain circular throughout the pairing process and the vorticity is uniform within the vortex cores.

The velocity, $\mathbf{V}_{\omega}$, of a vortex ring having circulation $\Gamma$ and radius $\sigma_{c}$ in the presence of another identical vortex ring and a background flow field is made up of three components:

$$
\mathbf{V}_{\omega}=\mathbf{V}_{\text {self }}+\mathbf{V}_{\text {ind }}+\overline{\mathbf{V}}\left(z_{c}, \sigma_{c}\right)
$$

where $\mathbf{V}_{\text {self }}$ and $\mathbf{V}_{\text {ind }}$ are the self-induced velocity and the velocity resulting from mutual induction, respectively. $z$ and $\sigma$ denote, respectively, the axial and radial coordinates in the near field. The subscript $c$ denotes a quantity associated with the vortex core centre. The background flow velocity $\overline{\mathbf{V}}(z, \sigma)$ varies from point to point in the flow field. This extra term, which has been ignored by researchers (for instance, Refs. 6 and 10) in the study of vortex pairing noise, gives a higher relative velocity between the vortex rings than those of $\overline{\mathbf{V}}$ $=0$. Thus it introduces the effect of background mean flow on vortex ring dynamics and their pairing noise. Denoting the core radius as $r_{c}$, the results of Kelvin and Stokes on velocity induction ${ }^{14}$ gives

$$
\mathbf{V}_{\text {self }}=\frac{\Gamma}{4 \pi \sigma_{c}}\left[\ln \left(\frac{8 \sigma_{c}}{r_{c}}\right)-\frac{1}{4}\right] \hat{z}
$$

and

$$
\mathbf{V}_{\text {ind }}=-\frac{\Gamma}{4 \pi} \oint \frac{\mathbf{y}-\mathbf{y}_{0}}{\left|\mathbf{y}-\mathbf{y}_{0}\right|^{3}} \times \frac{d \mathbf{y}_{0}}{d s} d s
$$

respectively, where $\hat{z}$ denotes an unit vector in the axial direction, and $d s$ an infinitesimal arc length on the second identical vortex ring. $\mathbf{y}$ and $\mathbf{y}_{0}$ are position vectors on the first and second vortex ring, respectively (Fig. 1). The integral in Eq. (2b) is taken over the whole periphery of the second vortex ring. Using the theory of Saffman, ${ }^{11}$ Leung et al. ${ }^{10}$ related $\Gamma$, $\sigma_{c}, r_{c}$, and the initial vortex ring core separation with jet velocity $W$, nozzle diameter $D$, and the Strouhal number

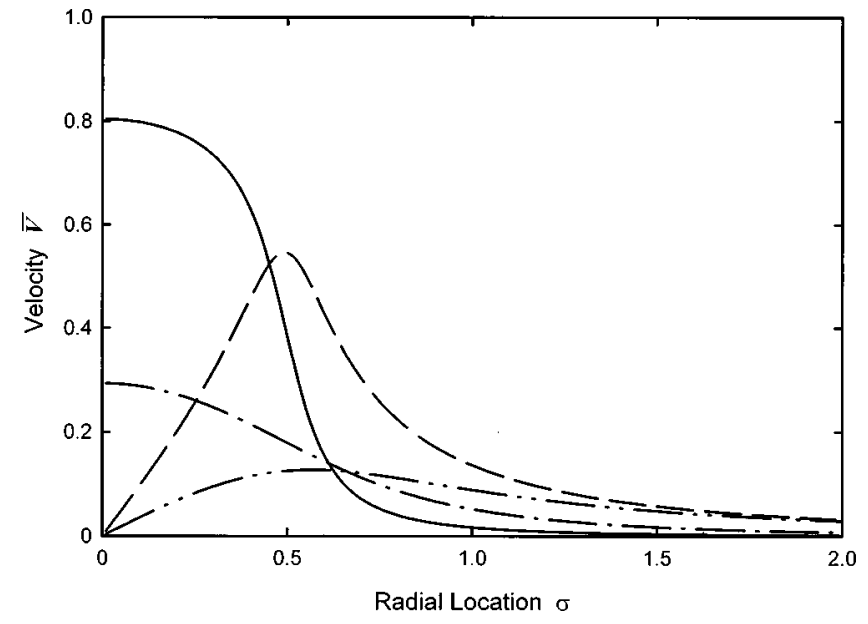

FIG. 2. Velocity profiles of present adopted background mean flow. Mean axial velocity at $z / D=0.1 ;-\ldots$ : mean axial velocity at $z / D=0.5$; — — : mean radial velocity at $z / D=0.1 ; \ldots \ldots$ : mean radial velocity at $z / D=0.5$.

$\mathrm{St}_{D}$. Details of the formulation have been given in Leung et al. ${ }^{10}$ and are not repeated here.

In the present study, unlike the case of Leung et al. ${ }^{10}$ the initial axial position, $z_{f}$, relative to the nozzle exit where a vortex ring is formed, is important because of the presence of a background mean flow velocity which varies from point to point in the flow field. The formation position of the vortex ring thus affects at least the initial separation of the vortex ring cores. Following the results of Saffman, ${ }^{11}$ it can be deduced that

$$
z_{f}=0.09 \pi^{-1 / 3} \mathrm{St}_{D}^{-2 / 3} D .
$$

The jet mean velocity profile given in Townsend ${ }^{15}$ cannot be used here to model the background mean flow $\overline{\mathbf{V}}(z, \sigma)$, as both the velocity induction laws of Stokes and Kelvin [Eq. (2)] and the vortex sound theory require divergence-free potential flow $^{16-18}$ while that given by Townsend, ${ }^{15}$ although it resembles the velocity profile of a practical jet, does not fulfill this criterion. The potential flow adopted in the present investigation is obtained using the method shown in Morse and Feshbach ${ }^{19}$ and the mean flow $\overline{\mathbf{V}}(z, \sigma)$ is given by

$$
\begin{aligned}
\overline{\mathbf{V}}(z, \sigma)= & \hat{z} \int_{0}^{\infty} W e^{-\alpha z} J_{1}(0.5 \alpha D) J_{0}(\alpha \sigma) d \alpha \\
& +\hat{\sigma} \int_{0}^{\infty} W e^{-\alpha z} J_{1}(0.5 \alpha D) J_{1}(\alpha \sigma) d \alpha,
\end{aligned}
$$

where $J_{0}$ and $J_{1}$ are the Bessel function of the first kind of the zero and first order, respectively. Equation (4) gives the axial velocity $W$ for $z=0, \sigma \leqslant 0.5 D$ and 0 for $z=0, \sigma$ $>0.5 D$. Details of the derivation are given in the Appendix. Some profiles of the mean flow $\overline{\mathbf{V}}(z, \sigma)$ are shown in Fig. 2. It can be observed that higher radial and axial velocity gradients appear close to the nozzle exit. Also, the initial growth rate of $\overline{\mathbf{V}}(z, \sigma)$ and the mean radial velocity are higher than those of low speed laminar or turbulent jets. ${ }^{20,21}$

The initial vortex ring core separation in the present study, unlike that in Leung et al., ${ }^{10}$ is found by solving the 
motion of the first vortex ring through integration of Eq. (1) with $\mathbf{V}_{\text {ind }}=0$ with respect to time, using the classical fourth order Runge-Kutta procedure. The length of the time integration, $t_{\omega}$, depends on the frequency or Strouhal number of vortex ring formation:

$$
t_{\omega}=\frac{D}{\mathrm{St}_{D} W} \text {. }
$$

The motions of the vortex rings during their interactions are also obtained by integrating Eq. (1) with respect to time, using the same numerical method. Since the present model allows an infinite number of slip-through processes which are hardly visualized in a real axisymmetric mixing layer, only the computed results within the first few slip-through processes will be discussed in detail.

Far field pressure fluctuations $p(\mathbf{x}, \tau)$, where $\mathbf{x}$ denotes a far field coordinate and $\tau$ the far field observer time, are calculated using the formula of Möhring: $:^{5}$

$$
p(\mathbf{x}, \tau)=\frac{\rho_{0}}{4 a_{0}^{2}|\mathbf{x}|^{3}} \sum \frac{d^{3}}{d t^{3}} \Gamma \sigma_{c}^{2} z_{c} \mathbf{x} \cdot\left(\hat{z} \hat{z}-\frac{1}{3}\right) \cdot \mathbf{x} .
$$

The sound field has the characteristics of a quadrupole. The involved time differentiations are carried out at the retarded time $t$, where $t=\tau-|\mathbf{x}| / a_{0}$. Since $\rho_{0}$ and $a_{0}$ are constants and $\mathbf{x}$ and $\hat{z}$ do not relate to sound generation mechanism, they are ignored in the foregoing discussions. The time steps for the differentiation and Runge-Kutta integration are so chosen such that a further halving of them does not give noticeable change in the computed results.

Investigations relating the mechanism of vortex pairing sound generation with the vortex ring motions have been carried out by the authors recently., ${ }^{9,12}$ Noting that the impulse, $I$, of a vortex ring is proportional to the product of circulation and the square of the vortex ring radius, $\Gamma \sigma_{c}^{2}$, Tang and $\mathrm{Ko}^{12}$ investigated the mechanism of thick core vortex ring pairing sound generation by decomposing the far field pressure fluctuations into four terms, each of which represents the sound generation by the coupling of a dynamic parameter, such as velocity or acceleration, with the time derivatives of $I$ :

$$
\begin{aligned}
p \propto & \sum I \frac{d^{3} z_{c}}{d t^{3}}+3 \sum \frac{d I}{d t} \frac{d^{2} z_{c}}{d t^{2}}+3 \sum \frac{d^{2} I}{d t^{2}} \frac{d z_{c}}{d t} \\
& +\sum z_{c} \frac{d^{3} I}{d t^{3}} .
\end{aligned}
$$

It should be noted that the right hand side of Eq. (7) is evaluated at retarded time. Equation (7) is obtained by a straightforward decomposition of Eq. (6), and thus is valid for both a thick core vortex ring and vortex filament pairing noise. The physical meanings of each of them can be found in Tang and $\mathrm{Ko}^{12}$ and are not repeated here. The changes of these four terms during pairing in the presence of a background potential flow of Eq. (4) are calculated. For simplicity, deformation of the vortex core is ignored. The method for changing the core sizes during vortex ring interaction follows that of Yamada and Matsui. ${ }^{13}$ For easy reference, the time derivatives on the right hand side of Eq. (7), namely the axial jerk $d^{3} z_{c} / d t^{3}$, the axial acceleration $d^{2} z_{c} / d t^{2}$, and the axial velocity $d z_{c} / d t$ are represented, respectively, by $J_{z}$, $A_{z}$, and $V_{z}$ in the foregoing discussion.

All computations were done using Microsoft FORTRAN PowerStation 1.0a on a 586 personal computer. Double precision calculation was adopted.

\section{RESULTS AND DISCUSSIONS}

In the foregoing discussion, all quantities, unless otherwise stated, are nondimensionalized. All lengths and time scales are normalized by the nozzle diameter $D$ and $D / W$, respectively. $W$ is the nozzle exit velocity. The present investigation aims at showing the trend rather than the exact values, and thus $D$ and $W$ are arbitrarily chosen to be unity and without unit, for simplicity. In the rest of the paper, the conditions of $\overline{\mathbf{V}} \neq 0$ and $\overline{\mathbf{V}}=0$ denote the cases with and without background mean velocity gradient, respectively. Also, the foregoing discussion is focused on the first three slip-through processes and $0.25 \leqslant \mathrm{St}_{D} \leqslant 4$, as $\mathrm{St}_{D}=0.25$ and 4 correspond roughly to the jet column mode and shear layer mode instabilities of a circular jet, respectively. ${ }^{7,20}$

\section{A. Vortex ring motions}

Without background mean velocity gradient, the motions of the inviscid vortex rings and the resulting far field pressure fluctuations are periodic. ${ }^{6}$ In the presence of the background potential flow, the vortex rings increase in diameter during their interaction, except for a short period just after their interaction commences. However, the patterns of their interactions depend significantly on $\mathrm{St}_{D}$. Figure 3(a) and (b) illustrates the locations of vortex ring core centers for $\mathrm{St}_{D}=0.25(\Delta t=0.4)$ and $4(\Delta t=0.02)$, respectively. The vortex ring core center locations for $\overline{\mathbf{V}}=0$ are included for reference. The numbers in these figures describe the sequence of the vortex ring core center motions. For all the $\mathrm{St}_{D}$ studied, the initially leading vortex ring has a larger diameter than the trailing one in the beginning of their interaction, owing to the radial velocity of the background flow field. The initial spacing between vortex rings is thus larger than that for $\overline{\mathbf{V}}=0$ for the same $\mathrm{St}_{D}$. It can be observed in Fig. 3 (a) that the trailing vortex ring first expands in diameter (at $z / D \approx 0.5)$. The decrease in the ring diameter that follows is probably due to the effect of the leading vortex ring which is then strong enough to counterbalance that of the background radial flow velocity. The major effect of the background mean flow is to increase the diameter of the initially leading vortex ring so that its diameter is larger than that of the initially trailing one at the beginning of the interaction. This is the result of the radial velocity component of the mean flow. The axial positions of the vortex cores are not affected by the mean flow as the latter is small at $z / D=0.5$, as shown in Fig. 2, and is even smaller for $z / D>1$. After the first slip-through process, which takes place at $z / D \approx 2.4$, the pattern of vortex ring interaction resembles that for $\overline{\mathbf{V}}=0$, except there is a slight increase in the radius of the vortex ring system centroid due to the background flow field. As shown in Fig. 3(b), for high $\mathrm{St}_{D}$, the much shorter vortex ring separation results in a more intense slip-through process, while the relatively weak vortex ring velocity induction cannot 


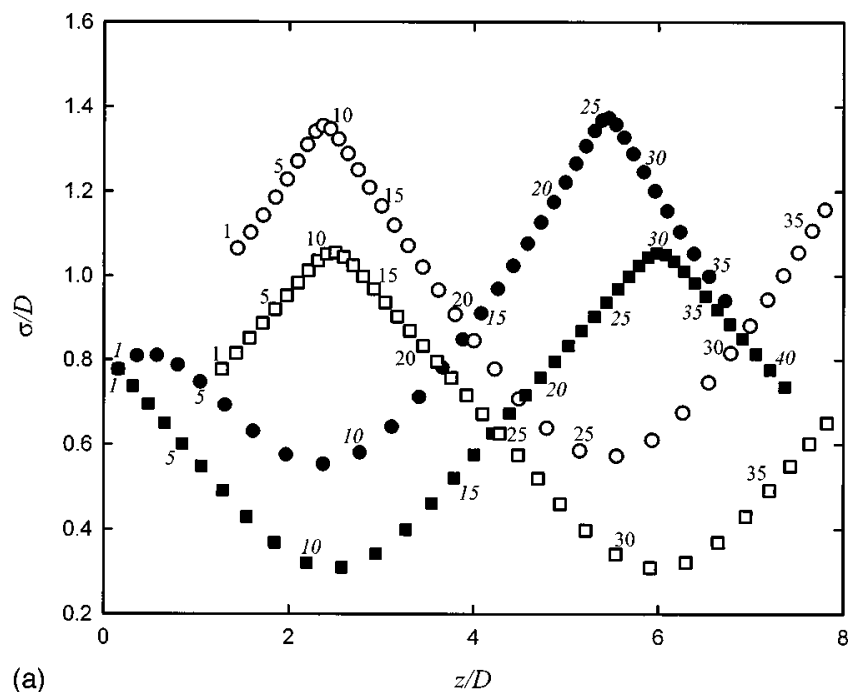

(a)

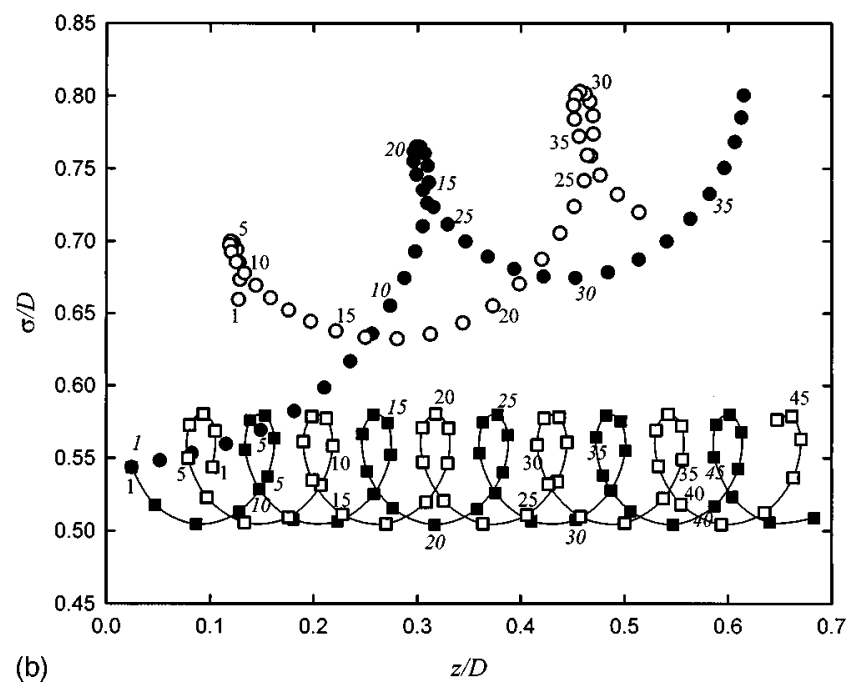

FIG. 3. Vortex ring interaction paths. (a) $\mathrm{St}_{D}=0.25$; (b) $\mathrm{St}_{D}=4$. $\bigcirc$ : Initially leading vortex ring $(\overline{\mathbf{V}} \neq 0)$; $\mathbf{0}$ : initially trailing vortex $\operatorname{ring}(\overline{\mathbf{V}} \neq 0)$. $\square$ : Initially leading vortex $\operatorname{ring}(\overline{\mathbf{V}}=0) ; \mathbf{Q}$ : initially trailing vortex $\operatorname{ring}(\overline{\mathbf{V}}$ $=0)$. Numbers describe vortex core center motion sequence: italic for initially trailing vortex ring; regular for initially leading vortex ring.

overcome the strong background radial velocity close to $\sigma / D=0.5$, so that no obvious shrinkage in the trailing vortex ring diameter can be observed for $z / D<0.2$. The backward axial motions of the vortex rings can also be observed for $\overline{\mathbf{V}}=0$ at this $\mathrm{St}_{D}$ [Fig. 3(b)]. However, the vortex ring motions become less unsteady in the presence of the background flow field [Eq. (4)]. This is a result of the increase in the initial spatial separation between the vortex ring cores. For other values of $\mathrm{St}_{D}$ studied the corresponding patterns lie between Fig. 3(a) and (b), and thus are not presented.

Figure 4 shows the time variation of the spatial separation of the vortex ring cores at $\mathrm{St}_{D}=1$ for both $\overline{\mathbf{V}} \neq 0$ and $\overline{\mathbf{V}}=0$. Actually, the higher the $\mathrm{St}_{D}$, the greater the initial rate of the decrease in this spatial separation. The presence of the background flow thus facilitates vortex ring coalescence. However, this spatial separation for $\overline{\mathbf{V}} \neq 0$ does not keep on decreasing. Its time variation pattern resembles that for $\overline{\mathbf{V}}$ $=0$, when the vortex rings are interacting in a region far

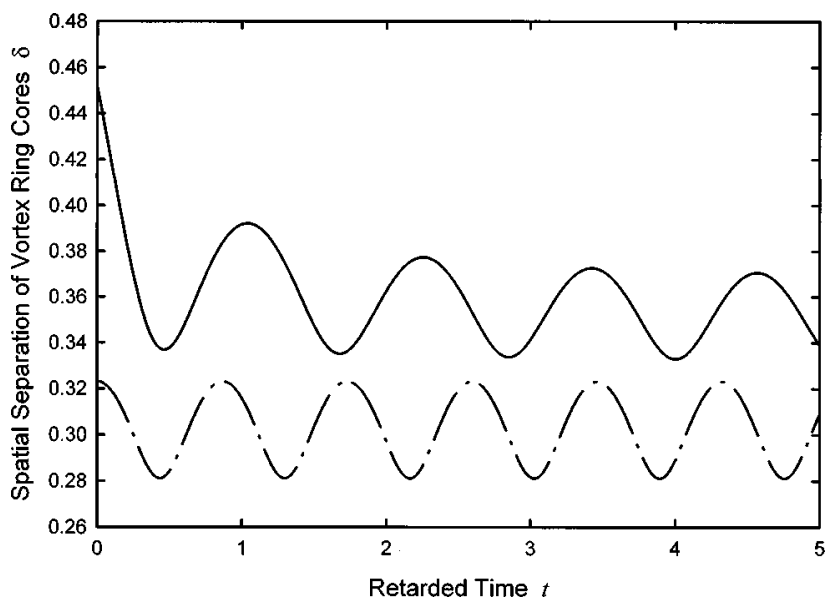

FIG. 4. Time variations of vortex ring spatial separation. $\mathrm{St}_{D}=1$. $\overline{\mathbf{V}} \neq 0 ;-\cdot-\overline{\mathbf{V}}=0$.

enough away from the nozzle where the background mean flow field becomes negligible when compared with that produced by the vortex rings.

It can be concluded that the background flow field does have significant effect on the details of the vortex ring interaction. The motion of the initially trailing vortex ring at small $z / D$ is especially affected by its presence.

\section{B. Pairing sound}

It is well known that the far field sound pressure fluctuations resulted from the vortex ring mutual slip-through process are periodic and their time variation patterns ${ }^{6,9}$ are similar for all $\mathrm{St}_{D}$. However, the latter depends on $\mathrm{St}_{D}$ in the presence of the background potential flow as defined by Eq. (4) and the far field pressure fluctuations are shown in Fig. 5. In Fig. 5, the solid lines represent the far field sound pressure fluctuations, while the other curves represent the four sound generation terms on the right hand side of Eq. (7). The major difference in the far field sound pressure fluctuations between the cases $\overline{\mathbf{V}} \neq 0$ and $\overline{\mathbf{V}}=0$ (see Fig. 6) is that there is a strong negative trough $P_{1-}$ in the beginning of the vortex ring interaction at different $\mathrm{St}_{D}$. This implies that in the presence of the background flow field the pressure fluctuations at this instant are also significant.

The variations of $P_{1-}$ and the first, second, and third pressure peaks $\left(P_{1+}, P_{2+}\right.$, and $\left.P_{3+}\right)$ with $\mathrm{St}_{D}$ are illustrated in Fig. 7. In the presence of the background flow there is a slight amplification of the sound radiated for $\mathrm{St}_{D}<0.6$, probably due to the effect of the additional vortex ring accelerations which compensate for the increased vortex ring core separation in the presence of the background flow field (Fig. 8). This will be discussed later. For $\mathrm{St}_{D}>0.6$, however, there is a significant reduction in the sound radiated due to the initial separation of the vortex ring cores is increased by the background flow field. The increase in vortex ring separation and the reduction of sound magnitude for $\mathrm{St}_{D}>0.6$ are similar to those of an uniform parallel external flow discussed by Leung et al. ${ }^{10}$ The amplitudes of all the far field pressure fluctuations obtained in the present study vary with the fourth power of $W$. This is the expected behavior of a quadrupole. 

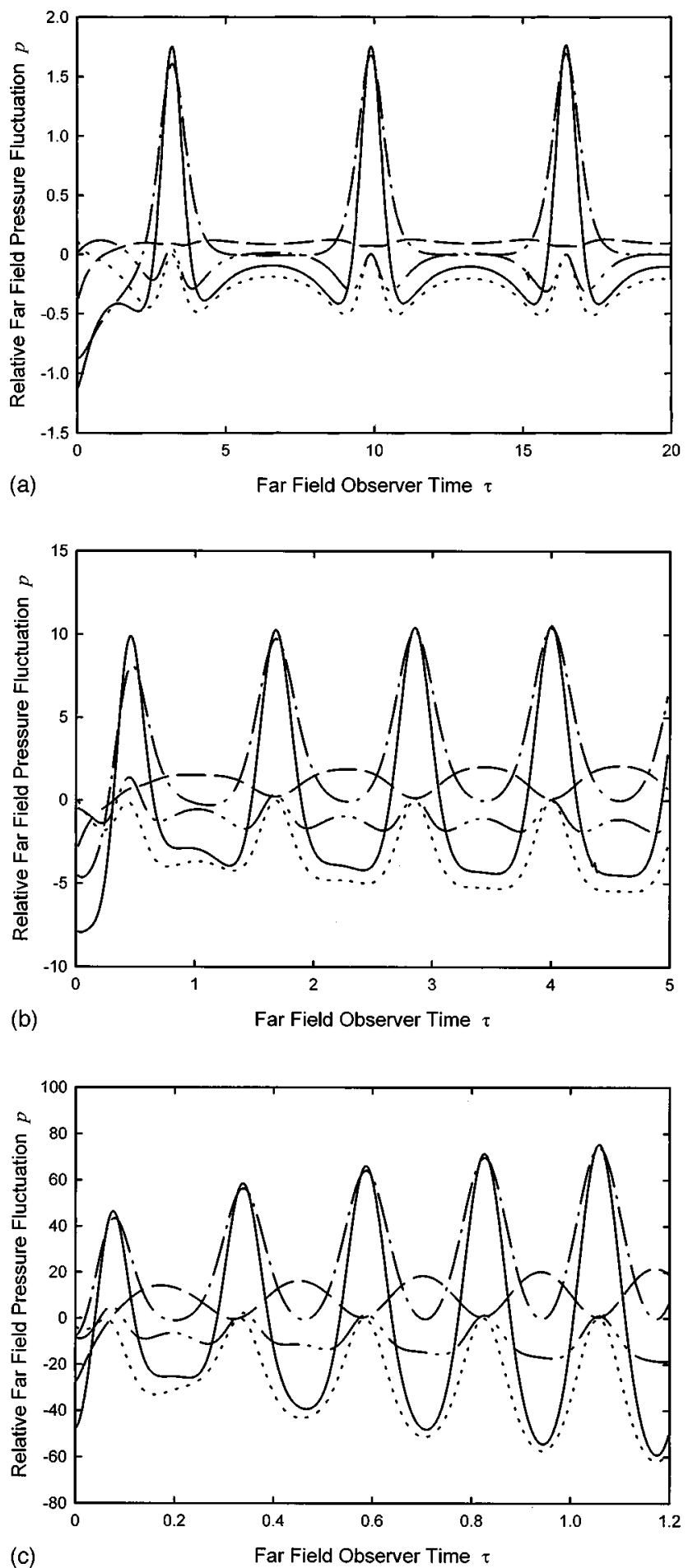

FIG. 5. Far field pressure fluctuations. (a) $\mathrm{St}_{D}=0.25$; (b) $\mathrm{St}_{D}=1$; (c) $\mathrm{St}_{D}$ =4. - — : Far field pressure fluctuations; - - $\Sigma I J_{z} ;-\cdots-$ : $3 \Sigma A_{z} d I / d t ;-.-: 3 \Sigma V_{z} d^{2} I / d t^{2} ; \cdots \cdots \cdot: \Sigma z_{c} d^{3} I / d t^{3}$.

Similar to the analysis of Tang and Ko, ${ }^{12}$ the far field pressure fluctuations are decomposed into four terms, as shown in Eq. (7). Their relative contributions at $\mathrm{St}_{D}=0.25$, 1 , and 4 are also shown in Fig. 5(a)-(c), respectively. The corresponding sound generation terms at $\mathrm{St}_{D}=0.25$ and $\overline{\mathbf{V}}$ $=0$ has been given in Fig. 6 . The foregoing discussions will be focused on the sound generation before the second slipthrough process as the subsequent far field pressure fluctuation pattern resembles those for $\overline{\mathbf{V}}=0$, which has been dis-

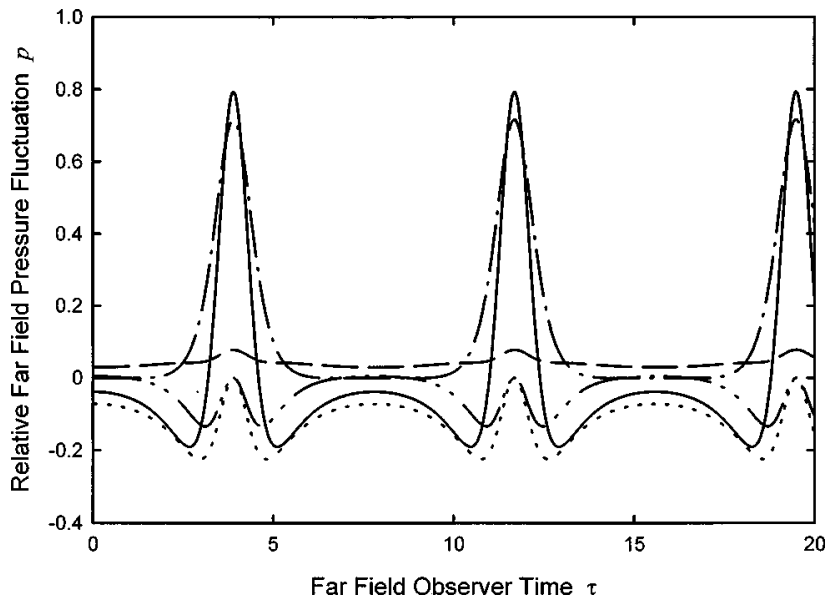

FIG. 6. Far field pressure fluctuations for $\overline{\mathbf{V}}=0 . \mathrm{St}_{D}=0.25$. Legends: same as those in Fig. 5.

cussed elsewhere. ${ }^{9,12}$ In the presence of the background flow, which is diverging at small $z / D, P_{1-}$ is mainly due to the terms $\Sigma I J_{z}$ and $\Sigma V_{z} d^{2} I / d t^{2}$, except at high $\mathrm{St}_{D}$ when the magnitudes of the other two terms become comparable to the latter. The term $\Sigma I J_{z}$, which corresponds to the sound generation through the vortex ring axial jerking motions, ${ }^{12}$ becomes more important in $P_{1-}$ as $\mathrm{St}_{D}$ increases. Similar for the case of $\overline{\mathbf{V}}=0$, the second term $\Sigma A_{z} d I / d t$ is of prime importance in the sound generation mechanism not at, or close to, the slip-through instants when the term $\sum V_{z} d^{2} I / d t^{2}$ dominates the sound radiation due to the high radial accelerations of vortex rings. ${ }^{12}$ It is also observed from Fig. 5 that the initial period of the negative $\Sigma I J_{z}$ increases with $\mathrm{St}_{D}$.

Thus the effect of the present background flow field on the sound generation is important, mainly in the beginning of the vortex ring interaction. The far field pressure time fluctuations after the first slip-through process, although of dif-

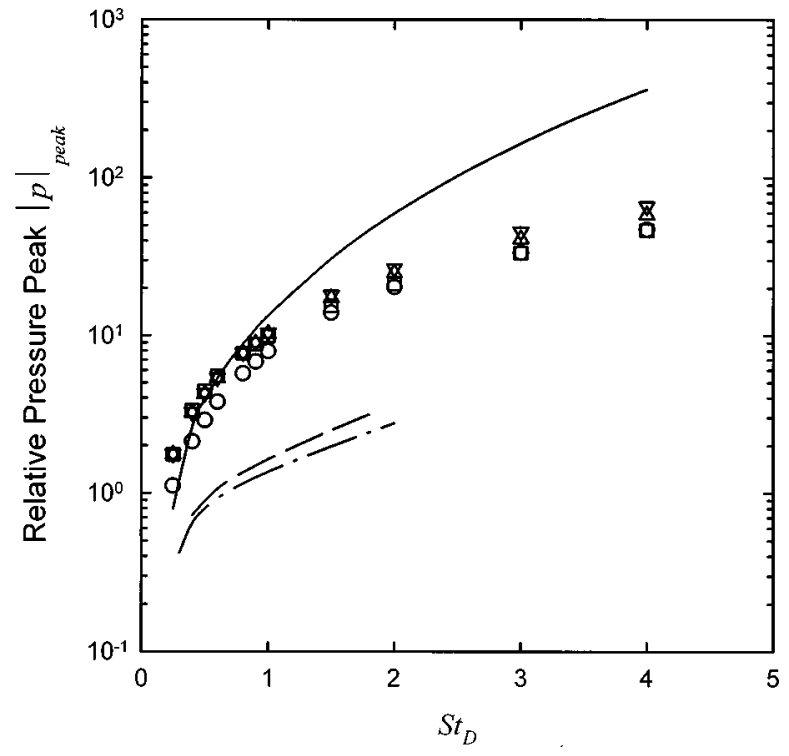

FIG. 7. Variations of pressure peaks with $\mathrm{St}_{D} \cdot \bigcirc:\left|P_{1-}\right| ; \square: P_{1+} ; \triangle$ : $P_{2+} ; \nabla: P_{3+} ;-\longrightarrow: \overline{\mathbf{V}}=0 ; \longrightarrow: \overline{\mathbf{V}}=0$ and $W_{0} / W=0.4$ (Ref. 10); - - $\overline{\mathbf{V}}=0$ and $W_{0} / W=0.5$ (Ref. 10). 


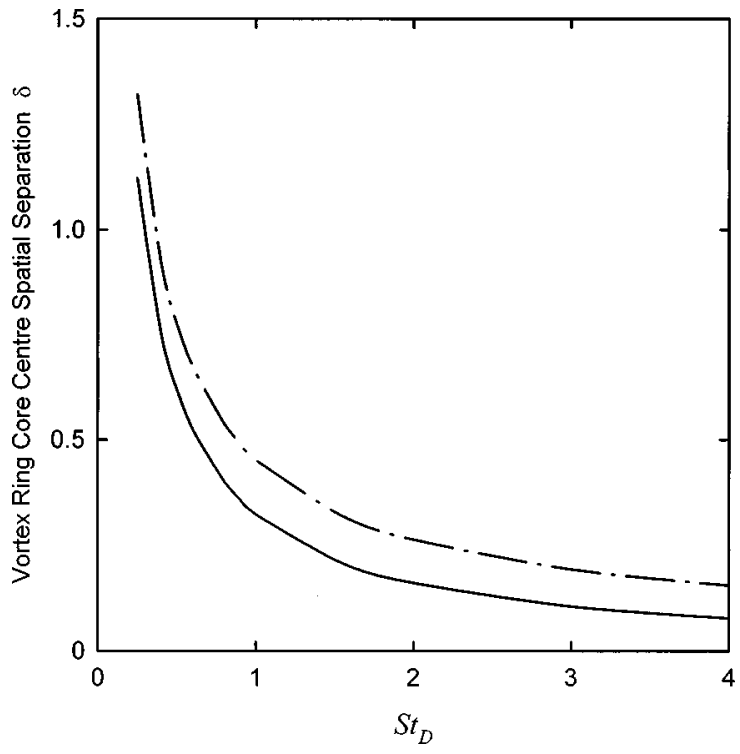

FIG. 8. Variations of initial vortex ring core separation with $\mathrm{St}_{D}$ $\overline{\mathbf{V}}=0 ;-\cdot-\overline{\mathbf{V}} \neq 0$.

ferent magnitudes, have time variation patterns similar to that for the $\overline{\mathbf{V}}=0$ (Fig. 6), implying similar sound generation mechanism in the two cases. The following discussions thus focus mainly on the initial sound generation process in which the vortex ring axial jerking motion and the second time derivative of vortex impulse are expected to be the major sources of sound.

It is noted from Fig. 5(a) that the term $\Sigma V_{z} d^{2} I / d t^{2}$ dominates the initial sound field at $\mathrm{St}_{D}=0.25$, but its dominance decreases as $\mathrm{St}_{D}$ increases, as shown in Fig. 5(b) and (c). As discussed in Tang and Ko, ${ }^{12}$ this term represents the generation of sound through the second time derivative of the vortex ring impulse as a vortex ring moving in constant velocity does not radiate sound. ${ }^{5}$ Since the vortex ring circulation is constant,

$$
\frac{d^{2} I}{d t^{2}}=\frac{d^{2}}{d t^{2}}\left(\Gamma \sigma_{c}^{2}\right)=2 \Gamma\left(V_{\sigma}^{2}+\sigma_{c} A_{\sigma}\right),
$$

but $V_{\sigma}^{2}$ is approximately one order lower than $\sigma_{c} A_{\sigma}$ in the beginning of the vortex ring interaction at low $\mathrm{St}_{D}$, as shown in Fig. 9(a) and (b). The dominance of the term $\Sigma V_{z} d^{2} I / d t^{2}$ in the sound generation process is then due to the radial accelerations of the vortex rings [Fig. 9(b)].

In the presence of a background flow field, the acceleration of a vortex ring depends on its velocity as well as the background flow velocity gradients in both the axial and radial directions:

$$
\frac{d^{2} \mathbf{y}_{c}}{d t^{2}}=A_{z} \hat{z}+A_{\sigma} \hat{\boldsymbol{\sigma}}=\frac{\partial}{\partial t} \mathbf{V}_{\text {ind }}+\frac{\partial}{\partial t} \mathbf{V}_{\text {self }}+\frac{d \mathbf{y}_{c}}{d t} \cdot \nabla \overline{\mathbf{V}} .
$$

However, using this decomposition of acceleration, it is difficult to extract one single term from the above equation to represent the total effect of the background flow on the vortex ring motions, as both $\mathbf{V}_{\text {self }}$ and $\mathbf{V}_{\text {ind }}$ depend on the locations of the vortex rings which are obviously functions of the background flow field. However, it is possible to study the contributions of each terms on the right hand side of Eq. (9)
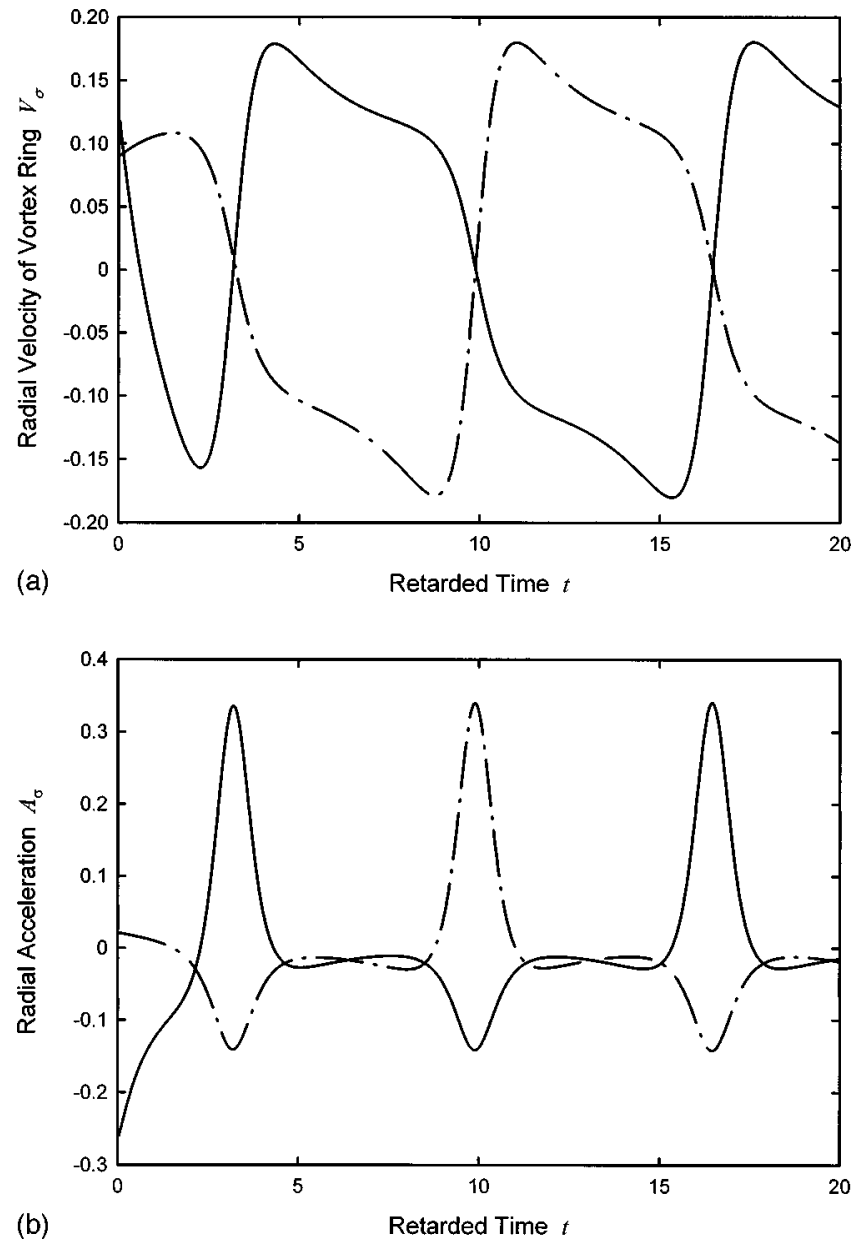

FIG. 9. (a) Time variations of vortex ring radial velocities. (b) Time variations of vortex ring radial accelerations. $\mathrm{St}_{D}=0.25$. — Initially trailing vortex ring; —.- : initially leading vortex ring.

in the overall radial acceleration of a vortex ring. The first term describes the acceleration due to mutual induction, the second term that due to self-induction, and the final one to the change in velocity directly due to the local background velocity field. The second term is not directly relevant to the vortex ring radial acceleration [Eq. (2b)]. From Fig. 10 the exceptionally high initial radial deceleration of the initially trailing vortex ring at $t=0$ mainly results from both the mutual induction and the radial background velocity field. The effect of mutual induction becomes dominant for $t>2$, when the vortex ring is moving in the region where the background flow velocity is small such that its effect on vortex ring motion is insignificant. Within this period of $t>2$, the major sound generation mechanism is due to the radial acceleration and is the same as that for $\overline{\mathbf{V}}=0$, although some contributions from the vortex ring axial jerking motions can be observed at the slip-through instants [Figs. 5(a), 6, and 9(b)]. It is also noted from Fig. 10 that the pattern of the time variation of the radial acceleration due to mutual induction resembles that for $\overline{\mathbf{V}}=0$ of Tang and Ko, ${ }^{9}$ but the magnitude of this acceleration for $\overline{\mathbf{V}} \neq 0$ in the beginning of the interaction is significant because of unequal vortex ring diameters [Fig. $3(\mathrm{a})$.

The initial radial deceleration of the initially trailing vortex ring becomes less dominant as a source of sound as $\mathrm{St}_{D}$ 


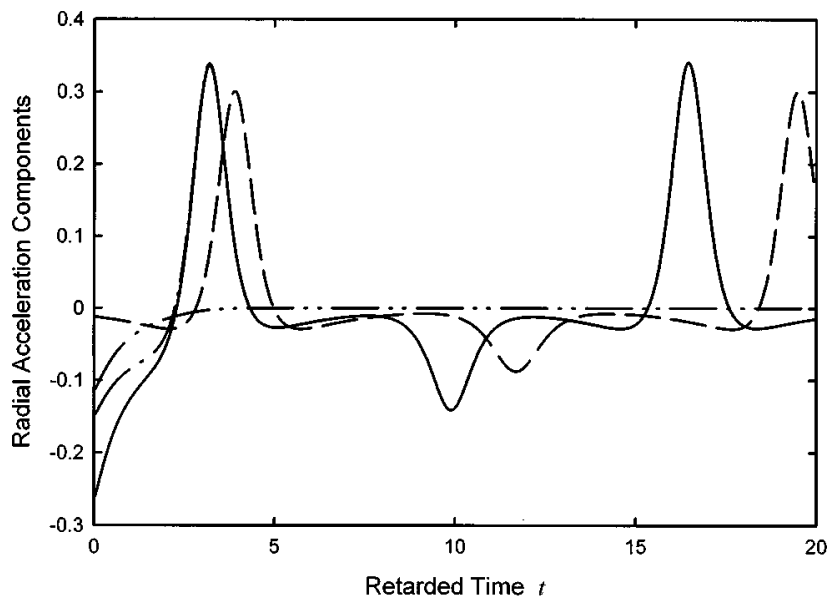

FIG. 10. Contributions of radial acceleration components for initially trailing vortex ring. $\mathrm{St}_{D}=0.25$. — : Total radial acceleration; —.—: mutual induction component; - -.- : direct contribution from background flow field; - - $\overline{\mathbf{V}}=0$.

increases. At high $\mathrm{St}_{D}$, the major sound generation mechanism at the initial stage of the interaction relates to the term $\Sigma I J_{z}$ [Fig. 5(c)]. Using the decomposition method of Eq. (1), the contribution of each of the velocity components in $\Sigma I J_{z}$ is summarized in Fig. 11. Again, the axial jerking motion due to the change in the local background flow velocity dominates the initial sound generation. This axial jerking motion is associated with the initially trailing vortex ring as shown in Fig. 12. The radial deceleration and the axial jerk $J_{z}$ of the initially trailing vortex ring remain the major sound generation mechanisms for the intermediate $\mathrm{St}_{D}$, and thus they are not discussed further. For all the $\mathrm{St}_{D}$ investigated, the initially leading vortex ring does not contribute much in the initial sound generation process as it is moving in a region where the background flow velocity and its gradient are small.

\section{Implication for low speed laminar jet noise generation}

The background flow field [Eq. (4)] adopted in the present study, while agreeing with the boundary condition of an inviscid circular jet, cannot really represent the jet because of the high initial growth rate and high radial mean velocity of the present adopted background flow field (Fig. 2). In a low speed laminar jet, the potential core extends to about four to five diameters downstream of the jet nozzle exit. ${ }^{20}$ Therefore, the duration of the background flow effect on vortex ring interaction, and thus the sound generation process, is prolonged. The results at high $\mathrm{St}_{D}$ in the present study are more relevant to a low speed laminar jet, where the vortex rings are interacting in a region with significant mean shear rate. ${ }^{22}$ The prominent peak in the far field pressure spectrum, close to that of the shear layer instability mode for a low speed laminar jet, ${ }^{7}$ is thus believed to be generated by the axial jerking and radial accelerating/decelerating motions of the vortex rings.

It is noted from Eq. (9) that the radial acceleration of a

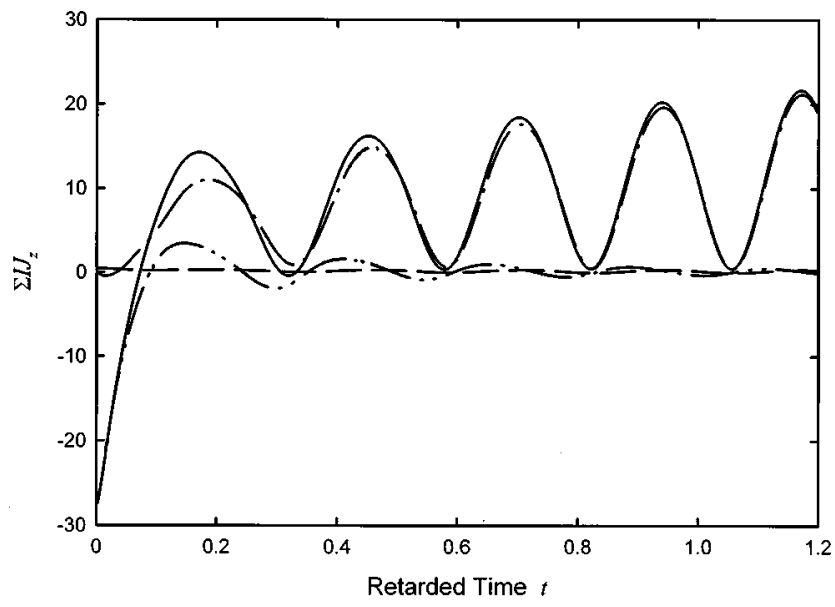

FIG. 11. Contributions of different axial jerk components in $\Sigma I J_{z} \cdot \mathrm{St}_{D}$ =4. ——: Total $\Sigma I J_{z} ;$ —. - : mutual induction component; —. - —: direct contribution from background flow field; - - : self-induction component.

vortex ring in the presence of the background flow is related to the background flow velocity gradient:

$$
\begin{aligned}
A_{\sigma} & =\hat{\sigma} \cdot \frac{d}{d t}\left(\mathbf{V}_{\text {ind }}+\overline{\mathbf{V}}\right) \\
& =\hat{\sigma} \cdot \frac{d}{d t} \mathbf{V}_{\text {ind }}+V_{z} \frac{\partial U_{\sigma}}{\partial z}+V_{\sigma} \frac{\partial U_{\sigma}}{\partial \sigma} \\
& =\hat{\sigma} \cdot \frac{d}{d t} \mathbf{V}_{\text {ind }}+V_{z} \frac{\partial U_{z}}{\partial \sigma}+V_{\sigma} \frac{\partial U_{\sigma}}{\partial \sigma},
\end{aligned}
$$

because $\overline{\mathbf{V}}$ is irrotational such that $\partial U_{\sigma} / \partial z=\partial U_{z} / \partial \sigma$. In the initial region of a shear layer, the mean shear rate is high due to the thin shear layer, while the radial mean velocity and its radial gradient are very small. Since the initial trailing vortex ring is propagating in the high speed side of the layer, its radial velocity depends significantly on the term $V_{z} \partial U_{z} / \partial \sigma$. The closer the vortex ring to the nozzle lip, that is the higher the Strouhal number, the more important this term becomes. Since $\partial U_{z} / \partial \sigma<0$ and the vortex rings are usually moving downstream, the mean shear rate tends to produce decelera-

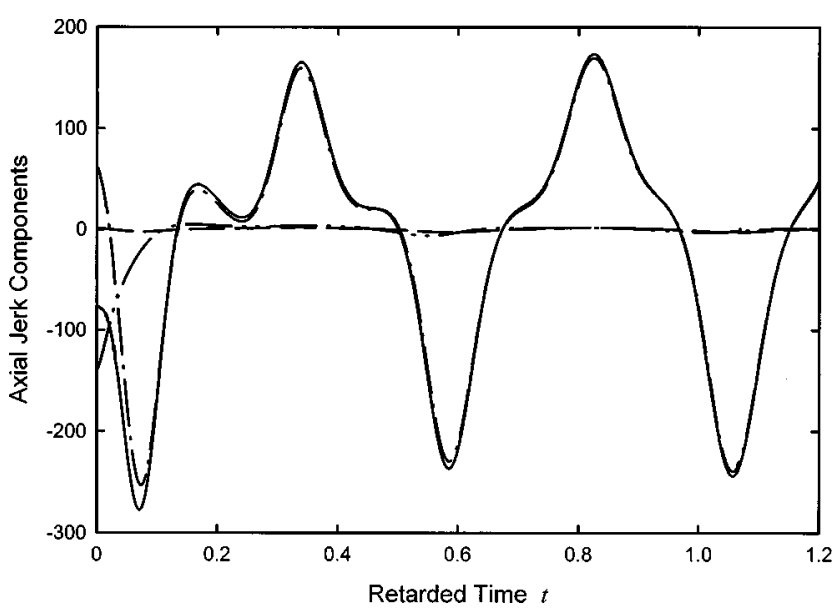

FIG. 12. Contributions of different velocity components in axial jerk of initially trailing vortex ring. $\mathrm{St}_{D}=4$. — duction component; - . - - direct contribution from background flow field; - -: self-induction component. 
tion. This appears consistent with the relatively high average deceleration of vortical structures measured by Tang and $\mathrm{Ko}^{9}$ for $z / D<0.5$. The mean shear rate is expected to play an important role in the sound radiation before and at the first slip-through instant of the high $\mathrm{St}_{D}$ initial vortex rings in a laminar shear layer.

Likewise, the axial jerk of a vortex ring in the presence of a background flow field can be decomposed as follow:

$$
\begin{aligned}
J_{z}= & \left(V_{z}^{2}-V_{\sigma}^{2}\right) \frac{\partial^{2} U_{z}}{\partial z^{2}}+A_{z} \frac{\partial U_{z}}{\partial z}+\left(A_{\sigma}-\frac{V_{\sigma}^{2}}{\sigma_{c}}\right) \frac{\partial U_{z}}{\partial \sigma}+\hat{z} \\
& \cdot\left(\frac{\partial^{2}}{\partial t^{2}} \mathbf{V}_{\text {self }}+\frac{\partial^{2}}{\partial t^{2}} \mathbf{V}_{\text {ind }}\right) .
\end{aligned}
$$

In a real jet, both $\partial^{2} U_{z} / \partial z^{2}$ and $\partial U_{z} / \partial z$ are small. The radius of the initial vortex rings is more or less the same due to the small shear layer thickness so that the radial acceleration of the trailing vortex ring is small. The corresponding velocity component is large, ${ }^{12}$ resulting in large initial negative axial jerk, as the fourth term on the right hand side of Eq. (11) is small when the coaxial vortex rings are of equal diameter. The effect of velocity induction due to vorticity has been shown to be of secondary importance in the total axial jerk of the vortex ring and in $\Sigma I J_{z}$ (Figs. 11 and 12). Since the growth rate of a low speed laminar jet is essentially lower than that of the background flow field adopted in the present study, it is expected that the axial jerking motion of the trailing vortex ring due to the mean shear rate is also important in the sound generation process at the slip-through instant in a low speed laminar jet. Therefore, the axial jerking motions and the radial accelerating/decelerating motions of vortex rings are believed to be the major sound generation mechanisms in a low speed laminar circular jet where a high mean shear rate exists. Also, the higher the mean shear rate, the higher is the sound level radiated. The present results therefore tend to suggest that the higher sound power of a conic nozzle than that of a large flanged one $\mathrm{e}^{23}$ is due to the higher mean shear rate in the conic nozzle shear layer.

Finally, it should be noted that the present vortex ring interaction model does not take into account the rolling-up process through which the vortex rings are formed. The reason is that in a low speed laminar jet, the shear layer instability near to the nozzle lip, from which the vortex rings are formed, is believed to be the source of the "excess" jet noise whose intensity varies approximately with the sixth power of the nozzle exit velocity. ${ }^{72}$ The corresponding sound field is not really a quadrupole, making Eq. (6) inapplicable.

\section{CONCLUSIONS}

In the present study, the aeroacoustics of vortex ring pairing in the presence of a background axisymmetric potential flow was investigated numerically using the velocity induction laws of Stokes and Kelvin. The background flow was obtained by solving the Laplace equation in cylindrical coordinates with the boundary condition of an inviscid circular jet. The dynamics of the vortex rings were also discussed.
Results suggest that the background flow field adopted for this paper substantially affects the initial sound generation process during the pairing of two vortex rings. Radial decelerating and axial jerking motions of the initially trailing vortex ring are the major sources of sound in the beginning of the interaction. The higher the Strouhal number, the more important the axial jerking motion in the sound generation process. At the slip-through instant, the radial acceleration of the trailing vortex ring becomes the major mechanism for sound generation.

The present results also show that the radial gradient of the background flow axial velocity, that is the mean shear rate, affects both the axial jerk and the radial acceleration/ deceleration of the vortex rings. High mean shear rate gives large radial deceleration and negative axial jerk. Since the shear rate in a low speed laminar jet is considerably higher than that of the present background flow, it is expected that higher sound levels are generated when the vortex rings are interacting within the region of high mean shear.

\section{APPENDIX: BACKGROUND POTENTIAL FLOW FIELD}

Let $\phi$ be the potential of the incompressible axisymmetric background flow field, thus

$$
\nabla^{2} \phi=\frac{\partial^{2} \phi}{\partial \sigma^{2}}+\frac{1}{\sigma} \frac{\partial \phi}{\partial \sigma}+\frac{\partial^{2} \phi}{\partial z^{2}}=0 .
$$

The simplest boundary condition for an inviscid circular jet being that for $z=0$,

$$
\left.\frac{\partial \phi}{\partial z}\right|_{z=0}= \begin{cases}W+W_{0}, & \text { for } \sigma \leqslant 0.5 D \\ W_{0}, & \text { for } \quad \sigma>0.5 D,\end{cases}
$$

where $W_{0}$ represents the velocity of a uniform flow external to the jet. Since $W_{0}$ has no effect on the vortex ring pairing noise as far as low Mach number flow is concerned, it is set to zero. It can be observed from Eq. (A1) that

$$
\nabla^{2}\left(\frac{\partial \phi}{\partial z}\right)=0
$$

The solution of Eq. (A3) with boundary condition (A2) can be obtained using the method shown in Morse and Feshbach ${ }^{19}$ which gives

$$
\frac{\partial \phi}{\partial z}=W \int_{0}^{\infty} e^{-\alpha z} J_{1}(0.5 \alpha D) J_{0}(\alpha \sigma) d \alpha .
$$

It is straightforward to show that the corresponding radial velocity is

$$
\frac{\partial \phi}{\partial \sigma}=W \int_{0}^{\infty} e^{-\alpha z} J_{1}(0.5 \alpha D) J_{1}(\alpha \sigma) d \alpha .
$$

Equations (A4) and (A5) also satisfy Eq. (A1). The flow field defined by Eqs. (A4) and (A5) is also irrotational.

\footnotetext{
${ }^{1}$ A. Powell, “Theory of vortex sound,', J. Acoust. Soc. Am. 36, 177-195 (1964).

${ }^{2}$ M. S. Howe, "Contributions to the theory of aerodynamic sound, with application to excess jet noise and the theory of the flute,' J. Fluid Mech. 71, 625-673 (1975).

${ }^{3}$ P. E. Doak, "Fluctuating total enthalpy as the basic generalized acoustic field,” Theor. Comput. Fluid Dyn. 10, 115-133 (1998).
} 
${ }^{4}$ C. D. Winant and F. K. Brownand, "Vortex pairing, the mechanism of turbulent mixing layer growth at moderate Reynolds number,' J. Fluid Mech. 63, 237-255 (1974).

${ }^{5}$ W. Möhring, "On the vortex sound at low Mach number,' J. Fluid Mech. 85, 685-691 (1978).

${ }^{6}$ T. Kambe and T. Minota, "Sound radiation from vortex systems," J. Sound Vib. 74, 61-72 (1981).

${ }^{7}$ J. E. Bridges and A. K. M. F. Hussain, "Roles of initial conditions and vortex pairing in jet noise," J. Sound Vib. 117, 289-311 (1987).

${ }^{8}$ J. Laufer and T. Yen, "Noise generation by a low-Mach-number jet,' J. Fluid Mech. 134, 1-31 (1983).

${ }^{9}$ S. K. Tang and N. W. M. Ko, "A Study on the noise generation mechanism in a circular jet," ASME Trans.: J. Fluids Eng. 115, 425-435 (1993).

${ }^{10}$ R. C. K. Leung, S. K. Tang, I. C. K. Ho, and N. W. M. Ko, "Vortex pairing as a model for jet noise generation,' AIAA J. 34, 669-675 (1996).

${ }^{11}$ P. G. Saffman, "The number of waves on unstable vortex rings,' J. Fluid Mech. 84, 625-639 (1978).

${ }^{12}$ S. K. Tang and N. W. M. Ko, "On sound generated from the interaction of two inviscid coaxial vortex rings moving in the same direction," J. Sound Vib. 187, 287-310 (1995).

${ }^{13} \mathrm{H}$. Yamada and T. Matsui, "Mutual slip-through of a pair of vortex rings,' Phys. Fluids 22, 1245-1249 (1979).
${ }^{14}$ H. Lamb, Hydrodynamics (Cambridge U.P., Cambridge, MA, 1993).

${ }^{15}$ A. A. Townsend, The Structure of Turbulent Shear Flow (Cambridge U.P., Cambridge, MA, 1976).

${ }^{16}$ P. G. Saffman, Vortex Dynamics (Cambridge U.P., Cambridge, MA, 1993).

${ }^{17}$ T. Kambe, "Acoustic emission by vortex motion," J. Fluid Mech. 173, 643-666 (1986).

${ }^{18}$ A. Powell, "Three-sound-pressures theorem, and its application, in aerodynamically generated sound,' J. Acoust. Soc. Am. 34, 902-906 (1962).

${ }^{19}$ P. M. Morse and H. Feshbach, Methods of Theoretical Physics (McGrawHill, New York, 1953).

${ }^{20}$ S. C. Crow and F. H. Champagne, "Orderly structures in jet turbulence," J. Fluid Mech. 48, 547-591 (1971).

${ }^{21}$ G. N. Abramovich, The Theory of Turbulent Jets (MIT, Cambridge, MA, 1963).

${ }^{22}$ K. B. M. Q. Zaman and A. K. M. F. Hussain, "Natural large-scale structures in the axisymmetric mixing layer,' J. Fluid Mech. 138, 325-351 (1984).

${ }^{23}$ J. Bridges and F. Hussain, "Effects of nozzle body on jet noise,' J. Sound Vib. 188, 407-418 (1995).

${ }^{24}$ D. G. Crighton, “The excess noise field of subsonic jets,' J. Fluid Mech. 56, 683-694 (1972) 